\title{
Photonic band structure and effective medium properties of doubly-resonant core-shell metallo-dielectric nanowire arrays: low-loss, isotropic optical negative-index behavior
}

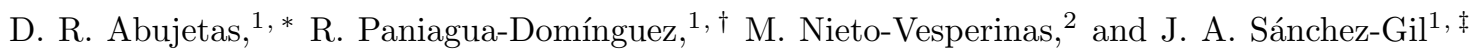 \\ ${ }^{1}$ Instituto de Estructura de la Materia (IEM-CSIC), \\ Consejo Superior de Investigaciones Cientificas, Serrano 121, 28006 Madrid, Spain \\ ${ }^{2}$ Instituto de Ciencia de Materiales de Madrid (ICMM-CSIC), \\ Consejo Superior de Investigaciones Científicas, Campus de Cantoblanco, 28049 Madrid, Spain
}

(Dated: October 8, 2018)

\begin{abstract}
We investigate theoretically and numerically the photonic band structure in the optical domain of an array of core-shell metal-semiconductor nanowires. Corresponding negative-index photonic bands are calculated, showing isotropic equifrequency surfaces. The effective (negative) electric permittivity and magnetic permeability, retrieved from S-parameters, are used to compare the performance of such nanowire arrays with homogeneous media in canonical examples, such as refraction through a prism and flat-lens focusing. Very good agreement is found, indeed confirming the effective medium behavior of the nanowire array as a low-loss, isotropic (2D) and bulk, optical negative index metamaterial. Indeed, disorder is introduced to further stress its robustness.
\end{abstract}

PACS numbers: 78.20.Ci,78.67.Pt,42.25.Bs,42.70.Qs

\section{INTRODUCTION}

Experiments in composite systems looking for a negative index of refraction (both permittivity and permeability being negative) at gigahertz frequencies ${ }^{1}$ were the first of this kind aiming at controlling the flow of electromagnetic waves; indeed, some fascinating properties have been since explored based on such composites 211 . However, to scale down these properties, not found in natural media, from the gigahertz regime to the optical domain, has posed a major challenge ${ }^{12,14}$, especially with regard to the negative magnetic permeability $15 / 16$ (negative electric response is trivial in the optical domain with metals). Despite some drawbacks of the original design, as for instance anisotropy, the initial philosophy to achieve this goal was to miniaturize the original ones, namely, split-ring resonators, magneto-dielectric elements, and related ${ }^{10 / 12 \mid 13}$. Nonetheless, the saturation of the magnetic response at high frequencies restricts this approach to the far infrared 15 . Other designs have been proposed that somehow mimic their behavior $\frac{12 \mid 17}{20}$, one of the most widely employed being metallic fishnet\$21. The emergence of ohmic losses in metals in the optical domain hinders also their performance.

To overcome this problem, various attempts have been made by exploiting magnetic resonances occurring in structures made of high-permittivity materials 22 , some of them combined with secondary plasmonic structures providing the electrical response $32-35$. Very recently, semiconductor nanostructures exhibiting magnetic resonances have been proposed as possible nonabsorbing metamaterial elements as well as emitters with unusual properties ${ }^{36-40}$. Actually, simple arrangements based on this idea have been proposed such as core-shell 3D and 2D metallo-dielectric nanostructures, respectively, nanospheres and nanowires, geometrically tuned so that their two first order Mie resonances (mag- netic and electric) spectrally overlap, which might play the role of doubly-resonant meta-atoms 4143 , directional scatterers4445, and transparent nanowires 464 48. Beside their robustness and isotropy stemming from their highly symmetric, single-structure meta-atoms, a theoretical figure of merit (f.o.m. $\left.=-\Re\left(n_{\text {eff }}\right) / \Im\left(n_{\text {eff }}\right)\right)$ of the order of f.o.m. $\sim 200$, has been reported in the case of core-shell nanowire (NW) arrays2. Incidentally, NW arrays have been proposed in turn as hyperbolic metamaterials 49 .

Nevertheless, a full description of their wave propagation properties must be done to determine whether their negative refraction behavior is a photonic-crystal diffraction effect that depends on the geometry sample, or a fully isotropic effective negative-index behavior (which we will referred to as negative-index metamaterial, NIM). In fact, it has been pointed out in Ref. 50 that the constitutive parameters retrieved through effective medium theories in other dielectric-rod ordered arrays do not reproduce the negative refraction observations in those composites, being mostly due to photonic crystal diffraction 5153. In this work, we thoroughly investigate the photonic band structure of an array of core-shell metal-semiconductor NWs, which was shown in Ref. 42 through parameter retrieval to exhibit low-loss, negative index behavior. This allows us to rigorously demonstrate (through equifrequency surfaces) and improve (fine tuning core-shell geometrical parameters) its highly isotropic character. Indeed, taking advantage of high isotropy and low losses, flat-lens focusing is presented through thick slabs with subwavelength resolution and dipole position independence, in good agreement with the expected behavior for a homogeneous slab with effective parameters as retrieved for the NW array. Limitations are also investigated with regard to image resolution, photonic-crystal surface termination, and disorder.

This paper is organized as follows. Corresponding negative-index photonic bands and equifrequency sur- 


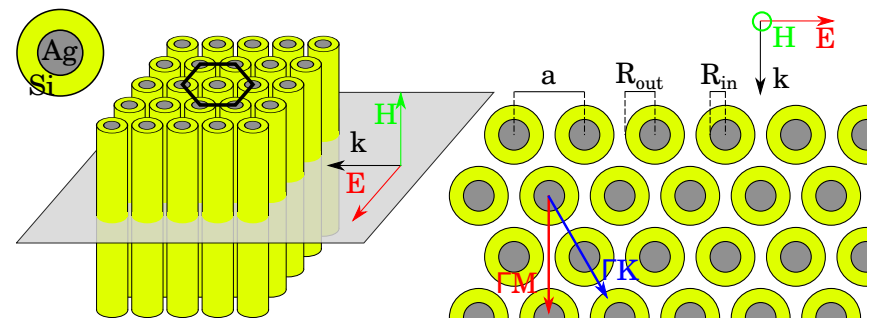

FIG. 1. (Color online) Schematic of the photonic crystal configuration under study, an hexagonal array of core-shell nanowires, including the relevant geometrical parameters. Only propagation in the plane perpendicular to the (infinitely long) NW axis will be considered with transverse electric polarization.

faces are calculated in Sec. II, confirming the effective medium behavior. In Sec. III, the performance of such NW arrays in the canonical example of flat-lens focusing through a thick slab, as compared with a homogeneous slab with the retrieved electric permittivity and magnetic permeability. This is also done for plane wave refraction through a prism in Sec. IV, introducing in turn disorder to support the robustness of the NW array as a low-loss optical NIM. Finally, Section V contains our concluding remarks.

\section{PHOTONIC BAND STRUCTURE}

First of all, we characterize the electromagnetic wave propagation in the $2 \mathrm{D}$ periodic composite medium consisting of core-shell metal-dielectric (infinitely long) NWs in a regular hexagonal array (see Fig. 1). For this purpose, we plot in Fig. 2 the 2D photonic band structure for the maximum symmetry directions for TE-polarized waves (magnetic field along the NW axis) of an hexagonal array with a lattice parameter of $a=350 \mathrm{~nm}$ made of Ag@Si core-shell NWs with external radius $R_{\text {out }}=$ $170 \mathrm{~nm}$, and inner radii $R_{\text {in }}=80,90 \mathrm{~nm}$. Details on the FEM numerical calculations of the photonic band structure are given in the appendix. Also included in Fig. 2 is the total Mie scattering efficiency of a single $\mathrm{Ag} @ \mathrm{Si}$ NW, obtained from Mie formulae for infinitely long coreshell cylinders ${ }^{54}$, explicitly plotting the contributions of the first three terms $\left(Q_{0}, Q_{1}, Q_{2}\right)$ stemming, respectively, from the zero-order magnetic, first-order (dipolar) electric, and second-order (quadrupolar) electric terms in the Mie multipolar expansion. The first one is due to a strong circulation of the electric displacement field inside the dielectric shell, so the position of the resonance mostly depends on the shell external radius. The $Q_{1}$ contribution corresponds to the localized surface plasmon resonance of the metal core. For obvious reasons, i.e. NIM behavior ${ }^{42}$, we choose the appropriate geometrical parameters so that both (electric and magnetic) resonances spectrally overlap, as clearly seen in Fig. 2; indeed, another inner radius $R_{\text {in }}=90 \mathrm{~nm}$ has been considered that slightly improves
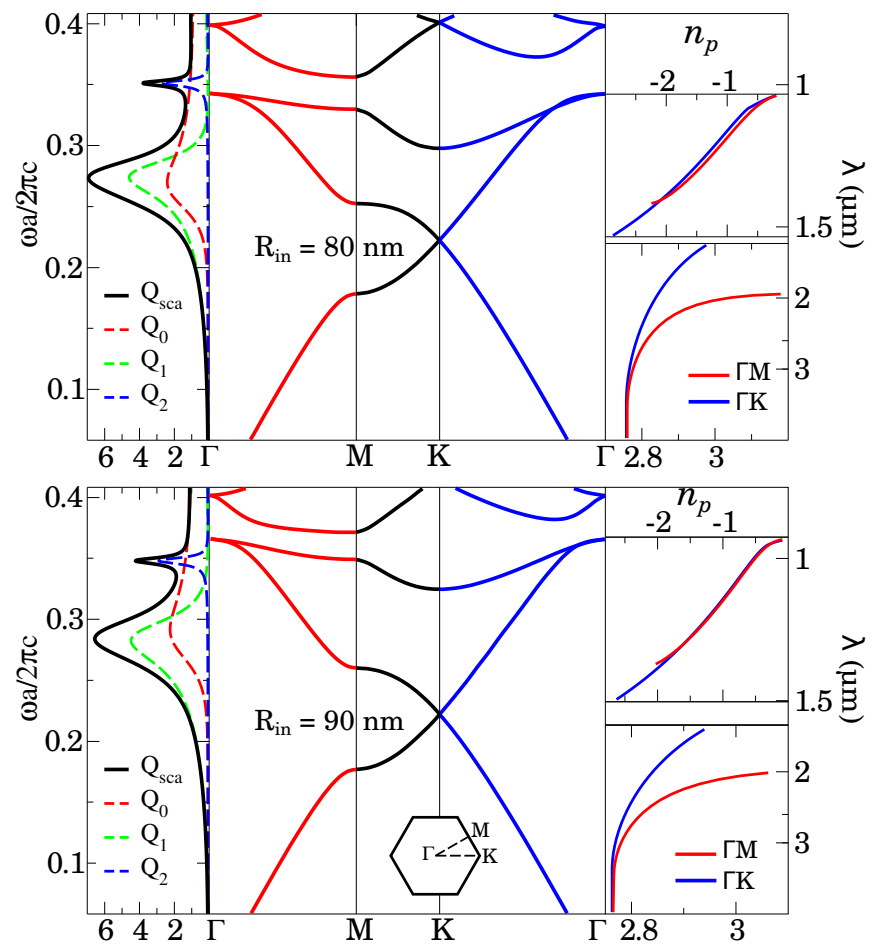

FIG. 2. (Color online) In-plane photonic band structure (center) of an hexagonal array ( $a=350 \mathrm{~nm}$ ) of $\mathrm{Ag} @$ Si core-shell nanowires with external radius $R_{\text {out }}=170 \mathrm{~nm}$ and internal radii: (top) $R_{\text {in }}=80 \mathrm{~nm}$ and (bottom) $R_{\text {in }}=90 \mathrm{~nm}$. In both plots: (left) The Mie scattering efficiency for an isolated nanowire is also shown (black curve), including separately the contributions from the first three multipolar terms (red, green, and blue curves); (right) phase index $n_{p}$ corresponding to the first two bands along the main $\Gamma \mathrm{M}$ (red curves) and $\Gamma \mathrm{K}$ (blue curves) directions.

such NIM behavior, as will be shown below. In addition, the lattice constant is such that the high filling fraction condition needed to achieve doubly negative effective parameters is fulfilled ${ }^{42}$; the lattice choice, hexagonal, favors this close packing and in turn isotropy.

The photonic bands present a partial gap in the $\Gamma \mathrm{M}$ direction in the first band and a full gap between the third and fourth bands. Besides, the slope of the second one is negative, and corresponds to a mode propagation with a negative phase velocity, as evidenced in the right graphs in Fig. 2, where the phase index $n_{p}$ corresponding to the first two bands along the main $\Gamma \mathrm{M}$ and $\Gamma \mathrm{K}$ directions is plotted. Furthermore, this band lies on the spectral region where the magnetic and electric resonances overlap, making it specially relevant to elucidate the effective NIM behavior stemming from strong individual electromagnetic responses of the isolated NWs. Thus let us study in detail this negative-phase second band in Fig. 2.

To this end, we represent in Fig. 3(a) the lowest four photonic bands, upon superimposing the curves at various propagation directions in between the maximum symmetry directions, $\Gamma \mathrm{M}$ and $\Gamma \mathrm{K}$ (red and blue curves, respectively), covering all relevant directions within the 


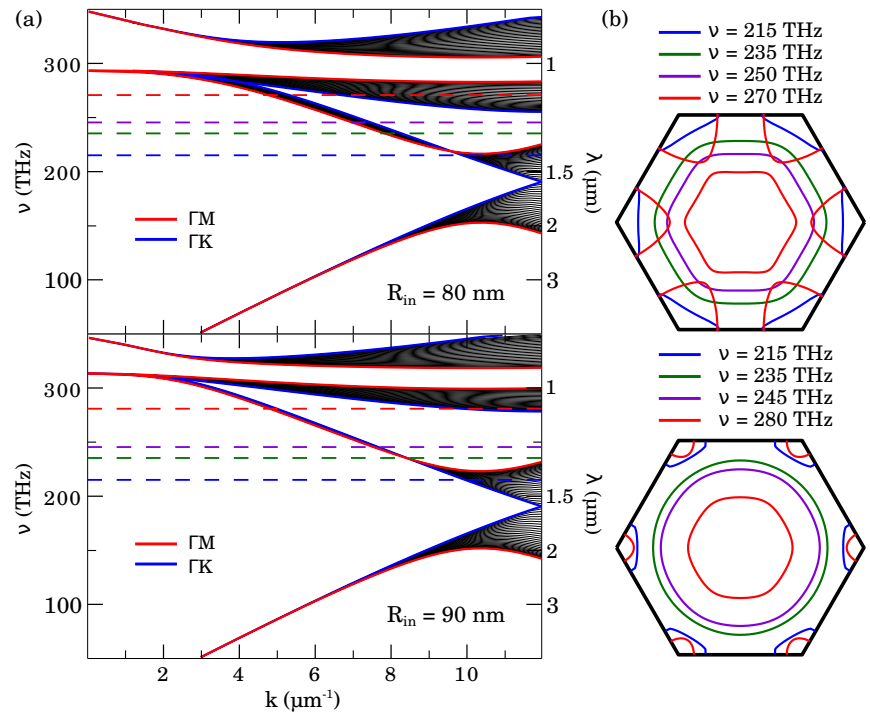

FIG. 3. (Color online) (a) Photonic band structure as in Fig. $2\left(a=350 \mathrm{~nm}, R_{\text {out }}=170 \mathrm{~nm}\right)$, but superimposing various (30) bands at different directions within the first Brillouin zone in between $\Gamma \mathrm{M}$ (red curves) and $\Gamma \mathrm{K}$ (blue curves). (b) Equifrequency surfaces for several frequencies (dashed horizontal lines in (a)) covering mainly the second band. In both $(\mathrm{a}, \mathrm{b}):\left(\right.$ top) $R_{\text {in }}=80 \mathrm{~nm}$ and (bottom) $R_{\text {in }}=90 \mathrm{~nm}$.

first Brillouin zone. In this manner, the partial gaps and the full gap at $300 \mathrm{THz}$ are clearly observed. More importantly, the nearly isotropic character of the second band is inferred, improving upon slightly increasing the NW inner radius from a dispersion of $10 \mathrm{THz}(\sim 5 \%)$ with wavevector direction shift for $R_{\mathrm{in}}=80 \mathrm{~nm}$, to a mere 2 $\mathrm{THz}(\sim 1 \%)$ for $R_{\text {in }}=90 \mathrm{~nm}$.

In order to clarify isotropy of the wave propagation properties in the second band we plot in Fig. 3(b) its equifrequency surfaces (EFSs) for several frequencies [horizontal lines in Fig. 3(a)], bearing in mind the hexagonal symmetry of the crystal. In the beginning of the second band, at $215 \mathrm{THz}(1.4 \mu \mathrm{m})$, we have the well known pockets due to the partial gap along the $\Gamma \mathrm{M}$ direction, whereas the only allowed propagation modes are close to the $\Gamma K$ directions. For frequencies above the partial gap [see Fig. 3(a)], all the mode propagation directions are again available and the EFSs become more circular, as shown at $235 \mathrm{THz}(1.28 \mu \mathrm{m})$ : Isotropy is indeed remarkable for the NW $R_{\text {in }}=90 \mathrm{~nm}$ inner radius at $245 \mathrm{THz}$ $(1.22 \mu \mathrm{m})$, with a nearly circular EFS. Then, as the frequency is further increased, at $270 \mathrm{THz}(1.11 \mu \mathrm{m})$ and at $280 \mathrm{THz}(1.07 \mu \mathrm{m})$, the EFSs of the second bands shrink and deform (become more hexagonal), overlapping with the third-band pockets appearing along the $\Gamma \mathrm{K}$ directions, thus breaking isotropy. Overall, it should be emphasized that there is a wide frequency range (215-260 $\mathrm{THz}$ ) in which the EFSs are almost circular (specially for $R_{\text {in }}=90 \mathrm{~nm}$ ) and the crystal might be described as an isotropic negative-index medium.

In the light of these results, it seems possible to make a

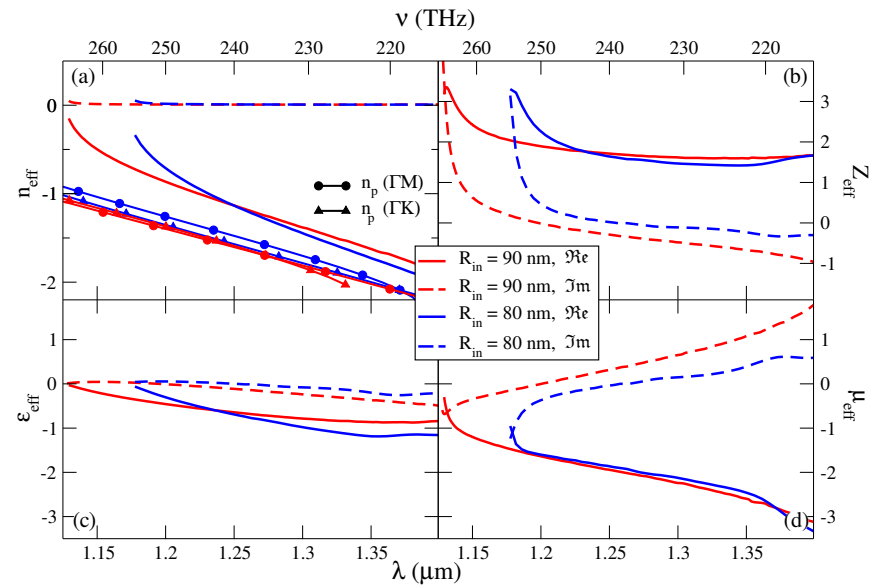

FIG. 4. (Color online) Spectral dependence (within the second NIM band) of the refractive index $n_{\text {eff }}$ (a), impedance $Z_{\text {eff }}$ (b), effective dielectric permittivity $\varepsilon_{\text {eff }}$ (c) and magnetic permeability $\mu_{\text {eff }}$ (d) retrieved from the S-parameters for Ag@Si core-shell nanowires, arranged in a hexagonal lattice with lattice parameter $a=350 \mathrm{~nm}$, with outer radius $R_{\text {out }}=170 \mathrm{~nm}$, and two inner radii: $R_{\text {in }}=80 \mathrm{~nm}$ (blue lines) and $R_{\text {in }}=90$ nm (red lines). Solid curves: real parts; dashed curves: imaginary parts. The phase index $n_{p}$ obtained from the photonic bands in Fig. 2 is also plotted (with symbols, top left).

fully isotropic 2D NW photonic crystal with negative index of refraction by taking advantage of both the negative index of the second band, due to the strong electromagnetic resonances of the isolated NWs, and of the isotropy that the photonic NW arrays shows within such band. To this aim, we first show in Fig. 4 the retrieved effective parameters (S-parameter retrieval procedur ${ }^{55566}$, through FEM calculations as detailed in the appendix) for the electric permittivity and magnetic permeability, along with the resulting index of refraction and impedance: The doubly negative behavior with $\Re\left(\varepsilon_{\text {eff }}\right), \Re\left(\mu_{\text {eff }}\right)<0$ within the NIM band is evident in Fig. 4. Low losses are also obtained: $\Im\left(\varepsilon_{\text {eff }}\right), \Im\left(\mu_{\text {eff }}\right) \sim 0$ [note that both might be slightly negative, in principle considered unphysical, but in practice as a result of the limitations of the parameter retrieval procedure with $\left.\Im\left(n_{\text {eff }}\right) \sim 0\right]$. The effective index is negative within the NIM band with nearly negligible losses: $\Re\left(n_{\text {eff }}\right)<0, \Im\left(n_{\text {eff }}\right) \sim 0$. Indeed, the retrieved refractive index for $R_{\text {in }}=90 \mathrm{~nm}$ yields an extremely large value of the f.o.m. $\sim 200$. Recall that the absorptive losses of a single core-shell NW are very small $\stackrel{42}{ }$, so that the remaining (small, but non-negligible) NIM losses are very likely due to scattering. The phase index $n_{p}$ obtained from the photonic bands in Fig. 2 is also plotted, in good agreement with $\Re\left(n_{\text {eff }}\right)$.

Incidentally, note that $\varepsilon_{\text {eff }}$ and $\mu_{\text {eff }}$ do not become -1 at the same frequency, which results in an impedance $Z_{\text {eff }} \equiv$ $\left(\mu_{\text {eff }} / \varepsilon_{\text {eff }}\right)^{1 / 2} \gtrsim 1$. Further tuning of the core-shell NW parameters could be done in order to achieve the ideal $Z_{\text {eff }}=1$; despite that, the resulting impedance is only slightly larger than 1 so that reflections at the interface 


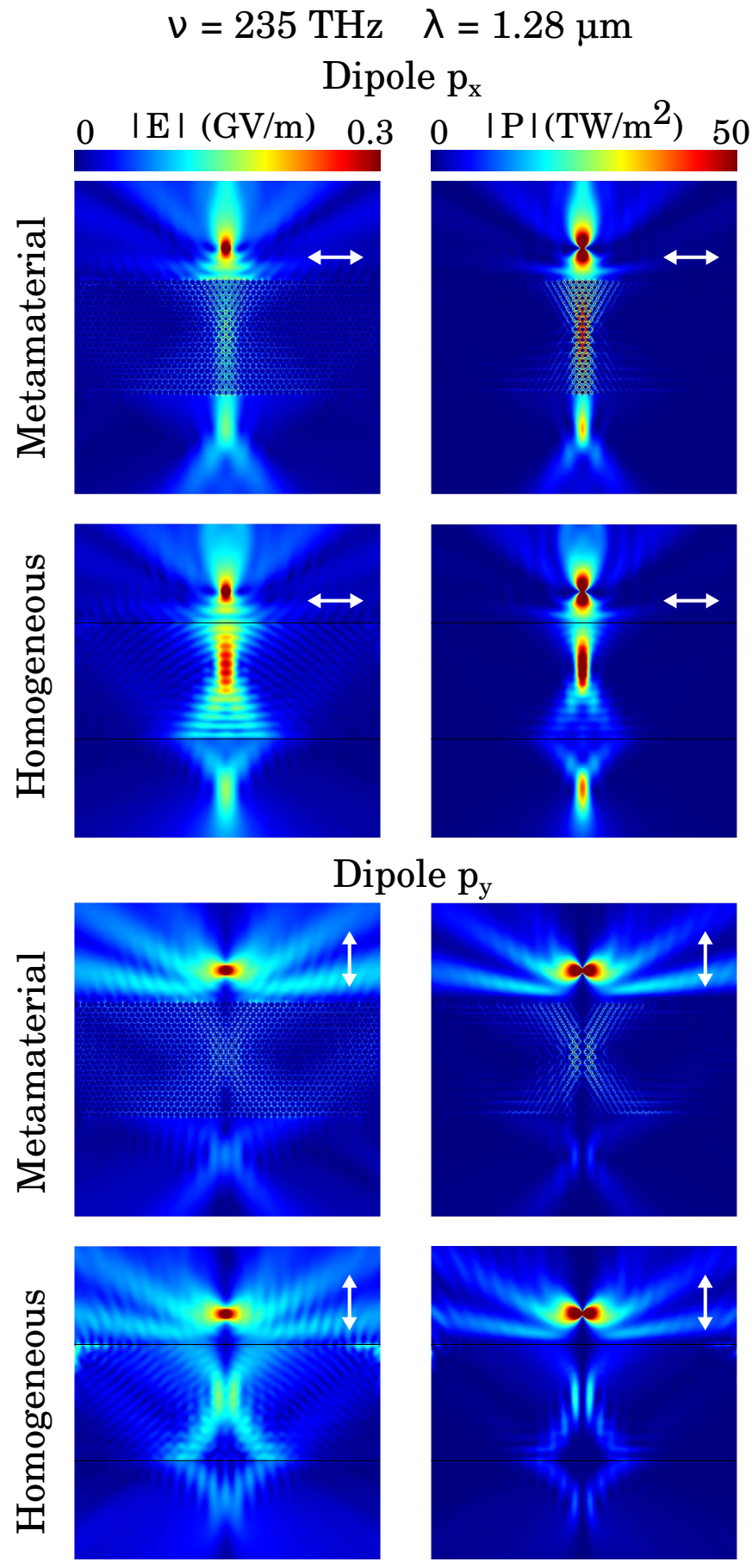

FIG. 5. (Color online) Transmission of the electromagnetic fields produced by an in-plane point dipole source at $235 \mathrm{THz}$ $(1.28 \mu \mathrm{m})$ located at $1.8 \mu \mathrm{m}$ above a slab made of $\mathrm{Ag} @ \mathrm{Si}$ core-shell nanowires with inner and outer radius $R_{\text {in }}=90$ $\mathrm{nm}$ and $R_{\text {out }}=170 \mathrm{~nm}$, respectively, arranged in a hexagonal lattice with lattice parameter $a=350 \mathrm{~nm}$ : Slab surface along the $\Gamma \mathrm{M}$ direction. Left: Norm of the in-plane electric field for a dipole parallel (top, $p_{x}$ ) and perpendicular (bottom, $p_{y}$ ) to the surface slab respectively. Right: Norm of the Poynting vector for a dipole parallel (top) and perpendicular (bottom) to the surface slab, respectively. The results for a homogeneous slab are also shown, with $\varepsilon_{\text {eff }}=-0.734-\imath 0.157$ and $\mu_{\mathrm{eff}}=-2.094+\imath 0.532$, so that $n_{\mathrm{eff}}=-1.264+\imath 0.0069$ (f.o.m. $\sim 180$ ) as obtained in Fig. 4 at $1.28 \mu \mathrm{m}$. are small, as will be shown below. However, at this point the important issue needs to be addressed whether or not this structure behaves as an isotropic homogeneous medium.

\section{FLAT-LENS FOCUSING: ISOTROPIC NIM BEHAVIOR}

To verify that the core-shell NW array presents an isotropic negative index of refraction, rather than considering plane wave negative refraction (which has been confirmed up to large angles of incidence in Ref. 42), we study the imaging of a point source through a slab, which no doubt places a more stringent test for it involves all plane wave angular components. We carry out numerical simulations of the transmission of the electromagnetic field radiated by an in-plane point dipole source at 235 $\mathrm{THz}(1.28 \mu \mathrm{m})$ placed in front of a finite $(22 \mathrm{NW}$ thick, namely, $6.7 \mu \mathrm{m}$ long) slab made of the structure studied in the previous section (see the appendix for the parameters of the FEM calculations); we focus on the $R_{\text {in }}=90$ nm case, which exhibits slightly higher isotropy. In Fig. 5 we plot the norm of the electric field (left column) and the Poynting vector (right column), respectively, for a dipole parallel to the surface of the slab (top), clearly revealing the effects of a medium that possesses a negative index of refraction. At the slab interfaces, light undergoes negative refraction and its field components are focused twice, one inside and other outside of the slab: The image produced on the other side of the slab presents similarities with the real one. For a dipole perpendicular to the slab (see Fig. 5 , bottom), again, we see the same phenomenology of negative refraction through the slab with the expected focusing effect for this dipole orientation. In addition, it is remarkable the fact that the Poynting vector is always zero along the propagation direction parallel to the dipole moment axis, so that light undergoes no significant spurious scattering losses when traveling through the photonic crystal; which in turn contributes to the effective medium parameters as losses, reportedly extremely small in this $\mathrm{NIM}^{\sqrt{42}}$, and thus in agreement with the above simulations.

Therefore the main features of flat lensing are fully retrieved $\sqrt[3|5| 6 \mid 14]{ }$. Furthermore, the agreement is remarkable with the results (shown also in Fig. 5) obtained upon considering an isotropic and homogeneous slab with effective parameters at $1.28 \mu \mathrm{m}$ given in Fig. 4 it should be emphasized that the (linear) color scale of both the electric field (left) and Poynting vector (right) norms are identical in all plots (columns), either for the NW array or for the homogeneous medium, and also that the scale is neither saturated nor altered within the slabs. Remarkably, note that very similar transmitted foci are produced: this is explicitly shown in Fig. 6, where the power transmitted at the image plane is presented for both the NW array and the homogeneous lenses. The fullwidth at half-maximum is of the order of $0.65 \mu \mathrm{m} \simeq \lambda / 2$, 


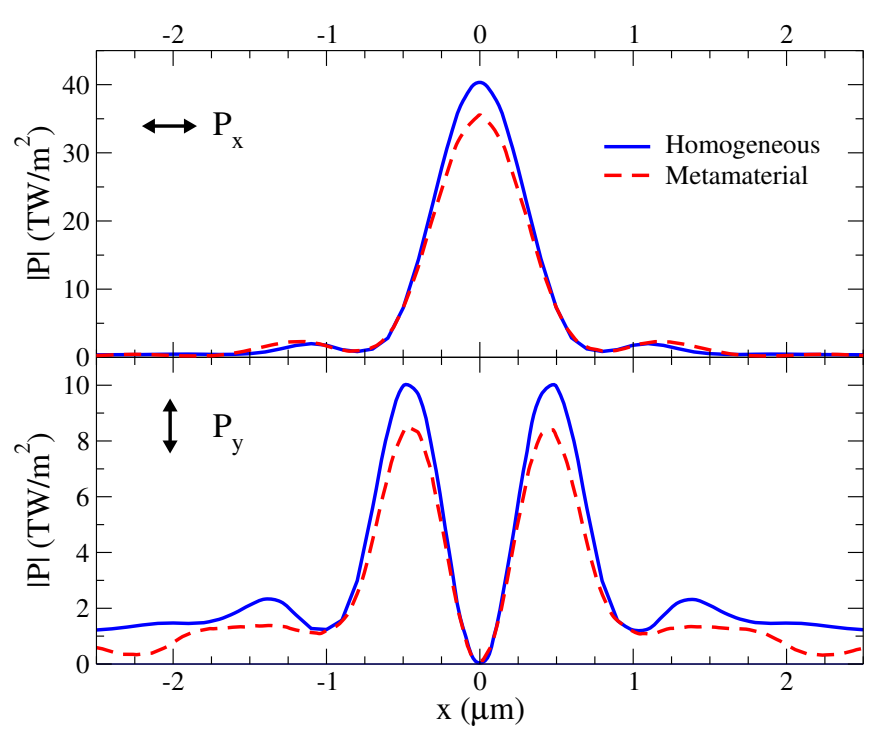

FIG. 6. (Color online) Poynting vector norms from Fig. 5 at the image plane.

yielding subwavelength resolution. Of course, since the source is not located deep in the near-field, superlensing in the proper sense $\mathrm{e}^{3}$ cannot be observed and resolution is diffraction limited (ultimately, resolution should be limited for the NW array to NW diameter). Overall, we clearly observe the phenomenology of (all-angle) negative refraction at the interface of the slab and the flatlens focusing process. This supports the highly isotropic, low-loss NIM behavior of the core-shell NW array, and the accuracy of the retrieved parameters, in turn ruling out photonic crystal collimation effects 5153 .

Nonetheless, we plot in Fig. 7 the EFSs for the particular case shown in Fig. 5. Momentum conservation along the impinging surface (wavevector component along $\Gamma \mathrm{M}$ ) is explicitly denoted by the green dashed line for a particular angle of incidence. Such plane wave component should be properly refracted with negative phase velocity (blue vector) and positive Poynting (red) vector. Since the EFS is nearly circular and larger than the air light cone, even plane wave components impinging from vacuum at grazing angles are negatively refracted. Note also that no additional diffracted components are expected. Therefore, all components of the dipole source should undergo negative refraction in a consistent manner; this corroborates the above presented dipole focusing through a slab (see Fig. 5).

Such NIM focusing behavior is in turn preserved upon moving the source. First, we show in Fig. 8 the negligible impact of shifting the dipole source (either $p_{x}$ or $p_{y}$ ) by half-period parallel to the surface, as compared to Fig. 5 and in agreement with the homogeneous slab, further ruling out any artifact induced by single metaatoms. Likewise, dipole positions are shifted perpendicular to the surface in Fig. 9 either closer (top) or farther (bottom); since this source displacement is more involved than the parallel one above, results are also included for

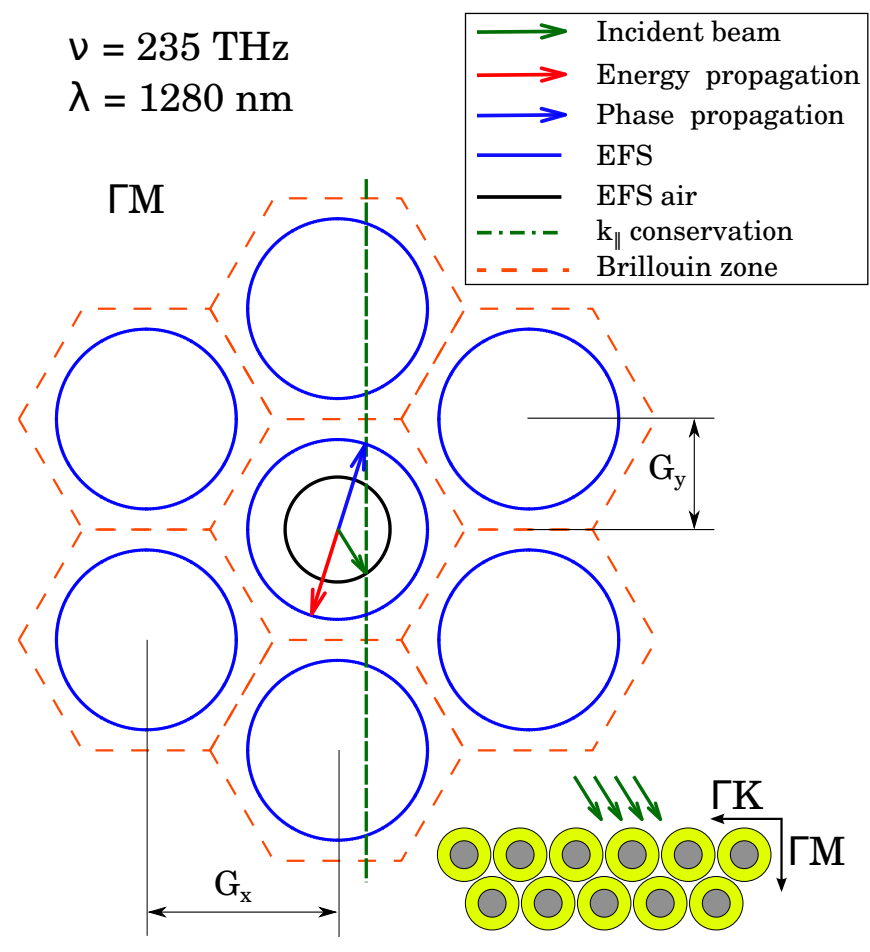

FIG. 7. (Color online) EFS at $235 \mathrm{THz}(1.28 \mu \mathrm{m})$ (blue circle) of a hexagonal lattice (lattice parameter $a=350 \mathrm{~nm}$, Brillouin zones delimited by red dashed hexagons) of Ag@Si core-shell nanowire with inner and outer radius $R_{\text {in }}=90 \mathrm{~nm}$ and $R_{\text {out }}=170 \mathrm{~nm}$. EFS of light impinging from vacuum/air is shown as a black circle. A dashed green (vertical) line denotes wavevector conservation along the $\Gamma \mathrm{M}$ direction for a particular angle of incidence, refracted accordingly as indicated by phase/energy (blue/red, respectively) vectors.

the homogeneous slab. According to the canonical NIM focusing effect (keeping the slab thickness fixed), if the dipole source is closer (respectively, farther) to the slab, the virtual image inside the slab gets closer (respectively, farther), whereas the image behind the slab appears farther (respectively, closer) from the slab surface; this is properly reproduced by the numerical calculations for the Ag@Si core-shell nanowire array.

Nonetheless, we would like to stress that the direction along which the array is terminated at the surface plays a crucial role despite isotropy. We calculate in Fig. 10 the dipole source transmission through a slab identical to that used in Fig. 5. except for the fact that the slab surface is along the $\Gamma K$ direction: it is evident that NIM focusing is remarkably worse. Two effects contribute to hinder it. First, surface roughness is larger along the $\Gamma \mathrm{K}$ direction, thus leading to larger scattering losses from single surface nanowires, as observed in Fig. 10 through both larger electromagnetic fields on the slab surface close to the source, and larger reflection losses. Second, momentum conservation parallel to the $\Gamma \mathrm{K}$ direction leads to a scenario different from that along $\Gamma M$ : apart from the expected negatively refracted beam, another refracted beam appears beyond a relatively small incidence angle 


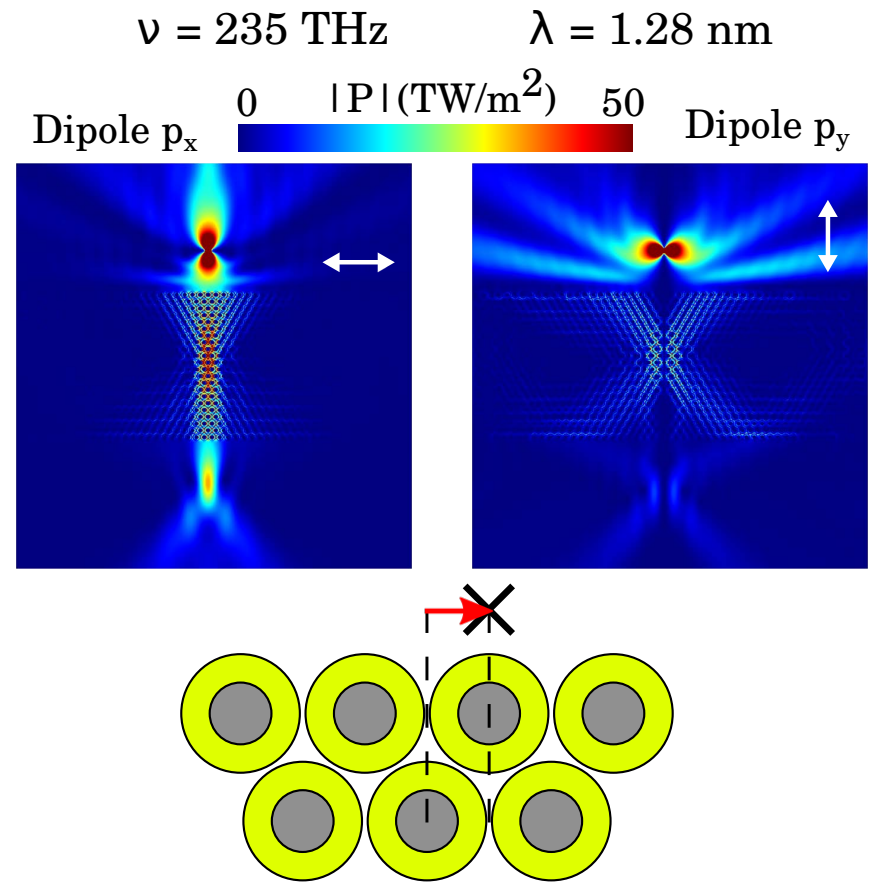

FIG. 8. (Color online) Top: Transmission through a slab made of a hexagonal array of Ag@Si core-shell nanowires $(a=$ $350 \mathrm{~nm}, R_{\text {in }}=90 \mathrm{~nm}, R_{\text {out }}=170 \mathrm{~nm}, \Gamma M$ surface direction), of the electromagnetic fields produced by an in-plane point dipole source at $235 \mathrm{THz}(1.28 \mu \mathrm{m})$, as in Fig. 5 (Poynting vector only), but for vertical/horizontal dipoles shifted halfperiod parallel to the slab surface as depicted (bottom). The results for the equivalent homogeneous slab are identical to those in Fig. 5 , and thus not shown.

as a diffracted beam at nearest Brillouin zone. This implies that dipole source components at a certain angle with respect to the surface normal exhibit two diffracted beams that preclude proper NIM focusing: such behavior is evidenced in Fig. 10 by crossing beams inside the slab.

\section{REFRACTION THROUGH A PRISM: TOLERANCE TO DISORDER}

In the preceding section we have demonstrated by means of flat-lens focusing that this doubly-resonant NW array behaves effectively as an actual isotropic (bulk) optical NIM when arranged in a close hexagonal lattice packet, stemming from the isotropic negative-phase behavior of the second photonic band. However, it has been shown that ordered configurations that exhibit a negative index of refraction may lose such effective property when the geometry is disordered. In this regard, it should be recalled that it has been shown in other cylinder arrays that Mie resonances (rather than Bragg scattering) govern in part the appearance of spectrally overlapping, photonic band gaps and higher-order bands $57 / 58$. Therefore, if Mie (rather than Bragg) scattering underlies the negative-index behavior, the latter should be more toler-

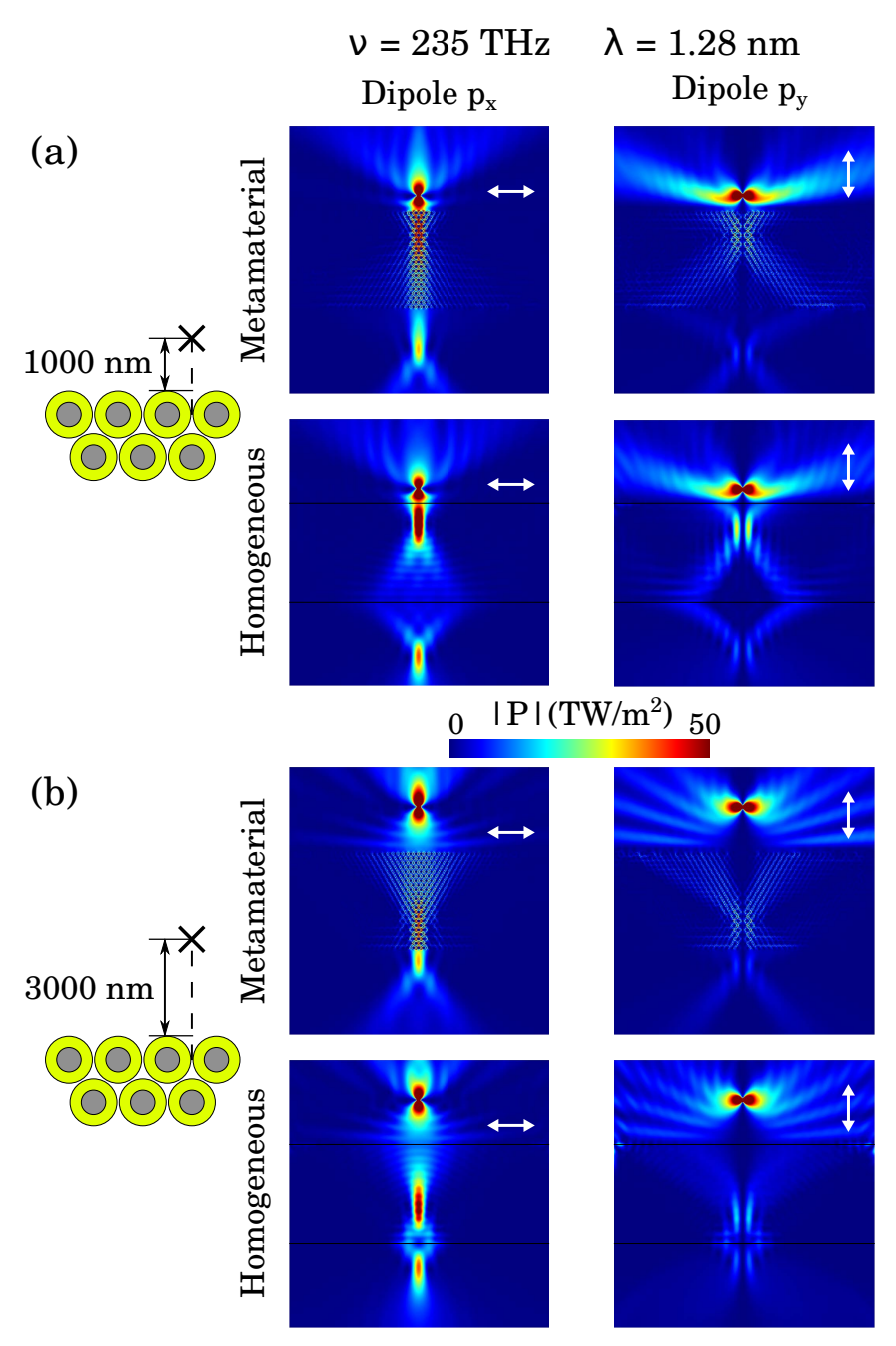

FIG. 9. (Color online) Transmission through a slab made of a hexagonal array of Ag@Si core-shell nanowires ( $a=350 \mathrm{~nm}$, $R_{\text {in }}=90 \mathrm{~nm}, R_{\text {out }}=170 \mathrm{~nm}, \Gamma M$ surface direction), of the electromagnetic fields produced by an in-plane point dipole source at $235 \mathrm{THz}(1.28 \mu \mathrm{m})$, as in Fig. 5 (Poynting vector only), but for horizontal (left column) and vertical (right column) dipoles shifted perpendicularly to the slab surface, both down (top) and up (bottom), as depicted by the left schemes. Results are also included for a homogeneous slab with $\varepsilon_{\text {eff }}=-0.734-\imath 0.157$ and $\mu_{\text {eff }}=-2.094+\imath 0.532$, so that $n_{\text {eff }}=-1.264+\imath 0.0069($ f.o.m. $\sim 180)$ as obtained in Fig. 4 at $1.28 \mu \mathrm{m}$.

ant to disorder than usual photonic crystals.

In order to show the robustness of doubly resonant NWs, let us show first in Fig. 11 the classical plane wave refraction through a rectangular prism with an angle of $\theta=30^{\circ}$, which actually involves two interfaces terminated in different photonic crystal directions (see the appendix for details of the FEM numerical calculations). We can see how the wavefronts leave the prisms above the normal, a clear signature of a NIM medium. Indeed, the full simulation of the wave propagation (see Video 1 in Ref. 42, Supplementary Information) revealed that 
$\nu=235 \mathrm{THz} \quad \lambda=1.28 \mu \mathrm{m}$
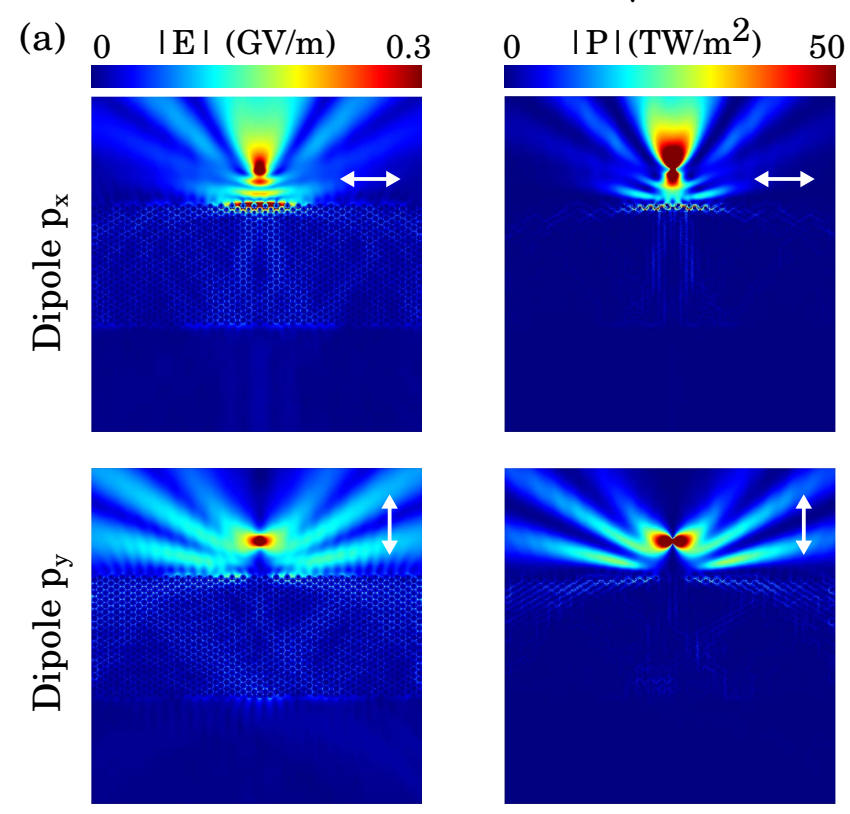

(b)

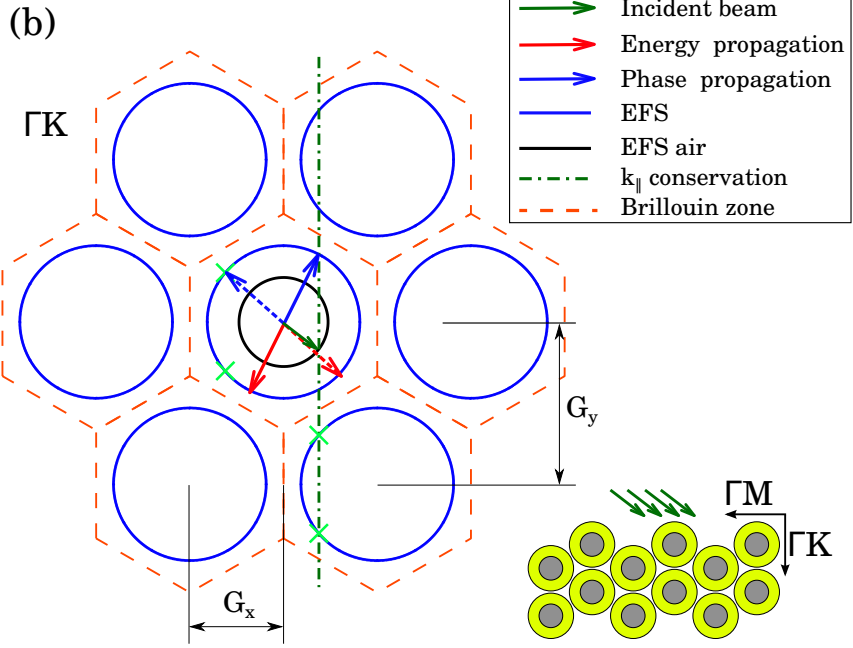

FIG. 10. (Color online) Top: Transmission through a slab made of a hexagonal array of Ag@Si core-shell nanowires $(a=$ $\left.350 \mathrm{~nm}, R_{\text {in }}=90 \mathrm{~nm}, R_{\text {out }}=170 \mathrm{~nm}\right)$, of the electromagnetic fields produced by in-plane point (horizontal/vertical) dipole sources at $235 \mathrm{THz}(1.28 \mu \mathrm{m})$, as in Fig. 5 . but for a slab surface along the $\Gamma \mathrm{K}$ direction. Bottom: Corresponding EFS (blue curves) of the hexagonal lattice along with that of light impinging from vacuum/air (black circle). A dashed green (vertical) line denotes wavevector conservation along the $\Gamma \mathrm{K}$ direction for a particular angle of incidence, producing accordingly two refracted beams as indicated by phase/energy (blue/red, respectively) vectors.

the wavefronts travel backward inside the prism (negative phase velocity), characteristic of a system having simultaneously negative permittivity and permeability. The homogeneous prism with the retrieved effective parameters nicely reproduces the expected negative refraction, thus further supporting the accuracy of the effective pa-
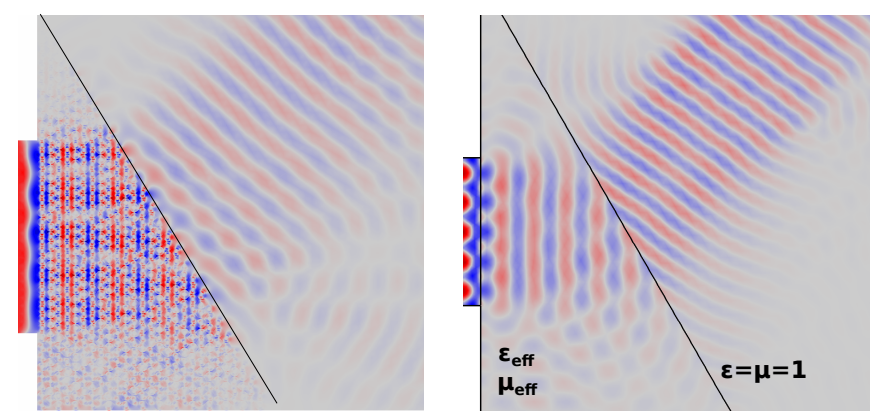

FIG. 11. (Color online) (a) Refraction through a $\theta=30^{\circ}$ prism made of a NIM built with Ag@Si core-shell NWs with $R_{\text {in }}=80 \mathrm{~nm}$ and $R_{\text {out }}=170 \mathrm{~nm}$ arranged in a hexagonal lattice $(a=350 \mathrm{~nm})$, illuminated by a transverse electric (truncated) plane wave $(1.215 \mu \mathrm{m})$ parallel to the left interface: contour maps of the out-of-plane, $z$-component of the magnetic field amplitude. (b) Same as in (a), but for a homogeneous medium with $\varepsilon_{\text {eff }}=-0.45+\imath 0.04$ and $\mu_{\mathrm{eff}}=-1.7-\imath 0.21$, so that $n_{\mathrm{eff}}=-0.88+\imath 0.01$ (f.o.m. $\left.\sim 80\right)$ as obtained in Fig. 4 .

rameters and the NIM behavior of the NW array.

We now introduce disorder in the prism geometry used in Fig. 11. In essence, some of the constituents are either shifted or removed from their original position in the ordered lattice. In Fig. 12(a) we explicitly show the NWs that have been shifted from their array positions in the prism and in Fig. 12(b) the plane wave propagation into the disordered prism. Although the wavefronts are somewhat perturbed by disorder upon crossing the prism and in the very near field region, we still observe the two key feature that characterize the negative index of refraction: an angle of transmission above the normal of the surface and a backward propagation of the wavefronts (negative phase velocity).

Moreover, such NIM features are preserved even if stronger scattering disorder is introduced, as shown in the lower panel in Fig. 12, where some NWs have been removed exactly along the wavefront propagation inside the prism. Actually, wave distortion is substantially larger than in the preceding case (shifted disorder), clearly observed in the near field close to the outgoing prism interface, although the backward propagation inside the prism and the (not so) far-field negatively refracted wavefronts are preserved. Bear in mind that, apart from positional disorder, if the NW filling fraction is substantially diminished, a decrease in the (absolute) values of the negative effective parameters is expected ${ }^{42}$.

Finally, disorder is also introduced in the slab geometry used in Fig. 5, again by shifting some of the NWs from their original position in the ordered lattice, as shown in Fig. 13. Note that, to make more relevant the impact of disorder, the shifted NWs are located in the central part of the slab, through which most of the dipole transmitted power is concentrated. A moderate amount of disorder (top) does not disturb the flat-lens focusing. Only when the number of shifted NWs increases (see Fig. 13, bot- 

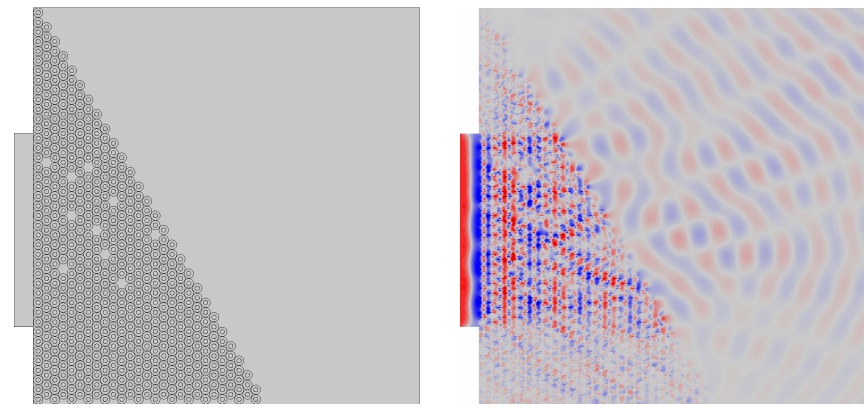

FIG. 12. (Color online) Negative refraction through disordered rectangular prisms with an angle of $\theta=30^{\circ}$ built with $\mathrm{Ag} @ \mathrm{Si}$ core-shell nanowires with $R_{\text {in }}=80 \mathrm{~nm}$ and $R_{\text {out }}=$ $170 \mathrm{~nm}$ arranged in a hexagonal lattice $(a=350 \mathrm{~nm})$ illuminated by a (transverse electric) plane wave parallel to the left interface at $1.215 \mu \mathrm{m}$. Disorder is introduced in two different manners: (top left) some of the NWs (darker) are shifted by an average of $1.5 \mathrm{~nm}$; (bottom left) some of the NWs are removed. Right column: Corresponding contour maps of the amplitude of the magnetic field (out-of-plane) component.

tom), the transmitted pattern is slightly altered, though the transmitted focal spot is still reproduced. Vacancy disorder (not shown here) is extremely sensitive in this case to the vacant position for obvious reasons, leading to strong scattering if placed nearby the intermediate focus region inside the slab, as expected. Nevertheless, we have shown that the properties of our closely packed, doublyresonant NWs are not only due to the periodicity of the lattice, but do survive even if substantial (either positional or vacancy-like) disorder is introduced, bearing in mind that filling fraction should be preserved.

\section{CONCLUDING REMARKS}

In conclusion, we have carried out a full description of the photonic band structure of doubly-resonant core-shell metallo-dielectric nanowire arrays, in order to demonstrate their behavior as low-loss, isotropic bulk optical NIMs, as reported in Ref 42 from the retrieved effective medium parameters. This must be done, as pointed out in Ref. [50, to rule out that their negative refraction behavior is mainly a photonic-crystal diffraction effect that depends on the specific geometry sample. The

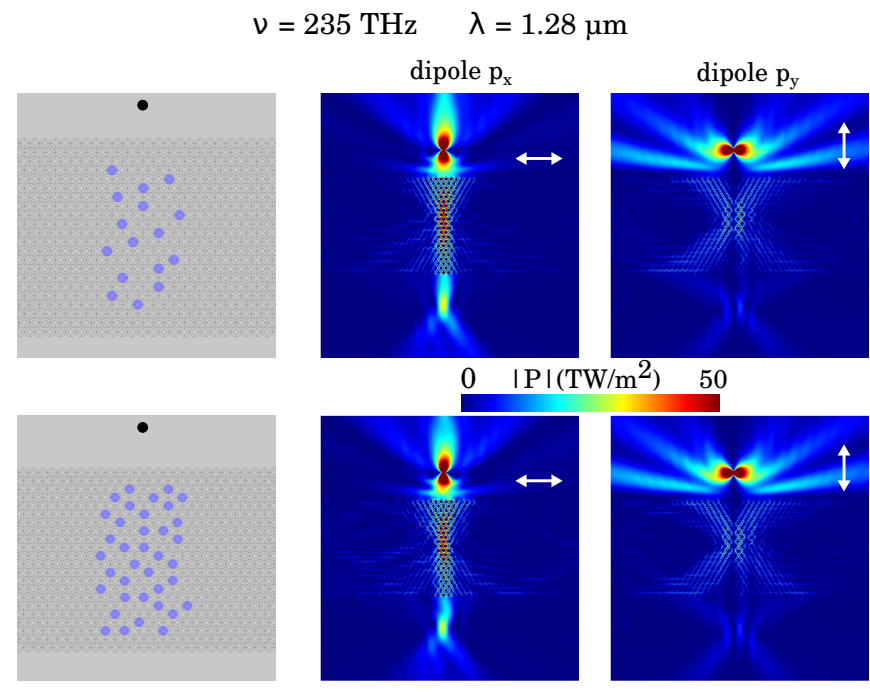

FIG. 13. (Color online) Transmission through a slab made of a hexagonal array of $\mathrm{Ag} @ \mathrm{Si}$ core-shell nanowires $(a=350 \mathrm{~nm}$, $R_{\text {in }}=90 \mathrm{~nm}, R_{\text {out }}=170 \mathrm{~nm}, \Gamma \mathrm{M}$ surface direction), of the electromagnetic fields produced by in-plane (horizontal, center, and vertical, right) point dipole sources at $235 \mathrm{THz}(1.28$ $\mu \mathrm{m}$ ), as in Fig. 5 (Poynting vector only), introducing disorder in the region below the dipoles by shifting the position of the NWs (darker ones) shown in the left column schemes: 16 (top row) and 37 (bottom row) NWs are randomly shifted.

2D photonic band structure has been presented, identifying the second photonic band with negative-index properties. The resulting EFSs in the first Brillouin zone are circular for most of this band, thus indicating the full (2D) isotropic character. In addition, such isotropic NIM behavior is very clearly evidenced through numerical simulations in canonical examples for finite geometries: Namely, point dipole transmission through a slab and negative refraction through a prism. Both cases are compared to the same configurations, but for a slab/prism characterized by homogeneous dielectric permittivity and magnetic permeability exactly given by the effective parameter retrieval (from S-parameters), in very good agreement. This further confirms the effective (highly isotropic) bulk NIM behavior, in turn explicitly manifesting the extremely low losses and suitable (close to 1) impedance. Finally, disorder is introduced in such finite prisms to support the robustness of the NW structures as NIMs, inferring that Mie resonances (rather than Bragg scattering) govern to a large extent their optical properties.

In this manner, we have rigorously demonstrated (and improved) the highly isotropic and ultra low-loss (f.o.m. 200) character of Ag@Si nanowire arrays, exhibiting indeed robust flat lensing with subwavelength resolution and dipole position independence, in good agreement with the expected behavior for a negativeindex homogeneous slab. Our results and procedure serve to fully assess such fascinating properties as optical NIMs of the doubly-resonant core-shell metallo-dielectric 
nanowire arrays, paving the way towards realistic fabrication thanks to the tolerance to specific geometric considerations and inhomogeneities.

\section{Appendix: FEM numerical calculations}

The numerical calculations of photonic band structures and wave propagation through slabs were carried out by means of full electromagnetic simulations using a Finite Element Method commercial software (COMSOL Multiphysics 4.4). For photonic band structures, an eigenfrequency study is applied to the unit cell upon Bloch boundary conditions to reproduce an infinite system. The calculated photonic band direction is determined by the periodicity of the $k$-vector.

In the case of the metameterial slabs, the simulated space consisted of an hexagonal array of of core-shell nanowires of height $H \approx 6.7 \mu \mathrm{m}$ and length $L \approx 35$ $\mu \mathrm{m}(22 \times 100$ nanowires), representing the slab, centered in a rectangle three times thicker than the slab and with the same length. In addition, all the physical system is surrounded by an additional layer of $2 \mu \mathrm{m}$ that is set as a perfectly matched layer (PML) to absorb all the outgoing radiation. The source of the electromagnetic field is simulated by an in-plane electric point dipole. For the homogeneous slab, the simulated space is the same as the metamaterial slab, but replacing the periodic array by a rectangle of the same $H$ and $L$, and applying a transition boundary condition to its boundaries, setting its thick- ness to $1 \mathrm{~nm}$ and its optical properties to those of the surrounding medium (air).

In all cases, silicon was assumed lossless in the frequency range studied, with a refractive index of $n_{S i}=3.5$ and silver material constant were taken from experimental values from 59 . The electromagnetic properties of the homogeneous medium were those determined by Sparameter retrieval procedure as in Refs. 5556 , and the rest of the space was set to be air $\left(n_{\text {air }}=1\right)$.

The meshing was done with the program built-in algorithm, which creates a triangular mesh. For photonic band structure calculations, the mesh maximum element size (MES) was set to $7.4 \mathrm{~nm}$ and the maximum element growth rate (MEGR) to 1.1. In addition, opposite edges of the unit cell were meshed identically by using the Copy Edge feature to ensure the symmetry of the system. For the transmission of the electromagnetic field radiated by a dipole, the MES was set to $100 \mathrm{~nm}$ and the MEGR to 1.3 .

\section{ACKNOWLEDGMENTS}

The authors acknowledge the Spanish "Ministerio de Economía y Competitividad" for financial support through the Consolider-Ingenio project EMET (CSD2008-00066), and NANOPLAS+ (FIS2012-31070), MINIELPHO (FIS2012-36113-C03-03) and FIS201455563-REDC.
* diego.romero@iem.cfmac.csic.es

$\dagger$ Present address: Data Storage Institute, Agency for Science, Technology and Research, 117608 Singapore.

$\ddagger$ j.sanchez@csic.es

1 R. Shelby, D.R. Smith, and S. Schultz, "Experimental verification of a negative index of refraction," Science 292, 77-79 (2001).

2 V. G. Veselago, "The electrodynamics of substances with simultaneously negative values of $\varepsilon$ and $\mu$," Sov. Phys. Usp. 10, 509-514 (1968).

3 J. B. Pendry, "Negative Refraction Makes a Perfect Lens," Phys. Rev. Lett. 85, 3966-3969 (2005).

4 N. García and M. Nieto-Vesperinas, "Left-Handed Materials Do Not Make a Perfect Lens," Phys. Rev. Lett. 88, 207403 (2002).

${ }^{5}$ E. Cubukcu, K. Aydin, E. Ozbay, S. Foteinopoulou, and C. M. Soukoulis, "Subwavelength Resolution in a TwoDimensional Photonic-Crystal-Based Superlens," Phys. Rev. Lett. 91, 207401 (2003).

6 A. Grbic and G. V. Eleftheriades, "Overcoming the Diffraction Limit with a Planar Left-Handed TransmissionLine Lens," Phys. Rev. Lett. 92, 117403 (2004).

7 S. A. Ramakrishna, "Physics of negative refractive index materials," Rep. Prog. Phys. 68, 449-521 (2005).

8 U. Leonhardt, "Optical Conformal Mapping," Science 312, 1777-1780 (2006).
9 J. B. Pendry, D. Schurig, and D. R. Smith, "Controlling electromagnetic fields," Science 312, 1780-1782 (2006).

10 R. Marqués, F. Martin, and M. Sorolla, Metamaterials with Negative Parameters: Theory, Design and Microwave Applications (Wiley, New York, 2007).

11 X. Zhang and Z. Liu, "Superlenses to overcome the diffraction limit," Nature Mater. 7, 435-441 (2008).

12 V. M. Shalaev, "Optical negative-index metamaterials," Nature Photon. 1, 41-48 (2007)

${ }^{13}$ C. M. Soukoulis and M. Wegener, "Past achievements and future challenges in the development of three-dimensional photonic metamaterials," Nature Photon. 5, 523-530 (2011).

14 T. Xu, M. Abashin, A. Agrawal, K. J. Chau, and H. J. Lezec, "All-angle negative refraction and active flat lensing of ultraviolet light," Nature 497, 470-474 (2013).

15 J. Zhou, Th. Koschny, M. Kafesaki, E. N. Economou, J. B. Pendry, and C. M. Soukoulis, "Saturation of the magnetic response of split-ring resonators at optical frequencies," Phys. Rev. Lett. 95, 223902 (2005).

16 A. Alú, A. Salandrino, and N. Engheta, "Negative effective permeability and left-handed materials at optical frequencies," Opt. Express 14, 1557-1567 (2006).

17 S. Zhang, W. Fan, N. C. Panoiu, K. J. Malloy, R. M. Osgood, and S. R. J. Brueck, "Optical negative-index bulk metamaterials consisting of $2 \mathrm{D}$ perforated metal-dielectric 
stacks," Opt. Express 14, 6778-6787 (2006).

18 J. Yao, Z. Liu, Y. Liu, Y. Wang, C. Sun, G. Bartal, A. M. Stacy, and X. Zhang, "Optical Negative Refraction in Bulk Metamaterials of Nanowires," Science 321, 930930 (2008).

19 J. Valentine, S. Zhang, T. Zentgraf, E. Ulin-Avila, D. A. Genov, G. Bartal, and X. Zhang, "Three-dimensional optical metamaterial with a negative refractive index," Nature 455, 376-379 (2008).

20 S. P. Burgos, R. de Waele, A. Polman, and H. A. Atwater, "A single-layer wide-angle negative-index metamaterial at visible frequencies," Nature Mater. 9, 407-412 (2010).

21 C. García-Meca, J. Hurtado, J. Martí, A. Martínez, W. Dickson, and A. V. Zayats, "Low-loss multilayered metamaterial exhibiting a negative index of refraction at visible wavelengths," Phys. Rev. Lett. 106, 067402 (2011).

22 M. I. Antonoyiannakis and J. B Pendry, "Mie resonances and bonding in photonic crystals," Europhys. Lett. 40, 613-618 (1997).

23 S. O'Brien, and J. B. Pendry, "Photonic band-gap effects and magnetic activity in dielectric composites," J. Phys.: Condens. Matter 14, 4035-4044 (2002).

24 M. S. Wheeler, J. S. Aitchison, and M. Mojahedi, "Threedimensional array of dielectric spheres with an isotropic negative permeability at infrared frequencies". Phys. Rev. B 72, 193103 (2005).

${ }^{25}$ L. Peng, L. Ran, H. Chen, H. Zhang, J. A. Kong, and T. M. Grzegorczyk, "Experimental observation of left-handed behavior in an array of standard dielectric resonators," Phys. Rev. Lett. 98, 157403 (2007).

26 J. A. Schuller, R. Zia, T. Taubner, and M. L. Brongersma, "Dielectric metamaterials based on electric \& magnetic resonances of silicon carbide particles," Phys. Rev. Lett. 99, 107401 (2007).

27 B. I. Popa, and S. A. Cummer, "Compact dielectric particles as a building block for low-loss magnetic metamaterials," Phys. Rev. Lett. 100, 207401 (2008).

${ }^{28}$ K. Vynck, D. Felbacq, E. Centeno, A. I. Căbuz, D. Cassagne, and B. Guizal, "All-dielectric rod-type metamaterials at optical frequencies," Phys. Rev. Lett. 102, 133901 (2009).

${ }^{29}$ Q. Zhao, J. Zhou, F. Zhang, and D. Lippens, "Mie resonance-based dielectric metamaterials," Mat. Today 12, 60-69 (2009).

30 J. Zhou, T. Koschny, M. Kafesaki, and C. M. Soukoulis, "Negative refractive index response of weakly and strongly coupled optical metamaterials," Phys. Rev. B 80, 035109 (2009).

31 E. F. Kuester, N. Memic, S. Shen, A. D. Scher, S. Kim, K. Kumley, and H. Loui, "A negative refractive index metamaterial based on a cubic array of layered nonmagnetic spherical particles," Progress In Electromagnetics Research B 33, 175-202 (2011).

32 V. Yannopapas, "Negative refraction in random photonic alloys of polaritonic and plasmonic microspheres," Phys. Rev. B 75, 035112 (2007).

33 A.-G. Kussow, A. Akyurtlu, and N. Angkawisittpan, "Optically isotropic negative index of refraction metamaterial," Phys. Status Solidi 997, 992-997 (2008).

${ }^{34}$ L. Jelinek and R. Marqués, "Artificial magnetism and lefthanded media from dielectric rings and rods," J. Phys.: Condens. Matter 22, 025902 (2010).

${ }^{35}$ L. Kang and D. Lippens, "Mie resonance based left-handed metamaterial in the visible frequency range," Phys. Rev.
B 83, 195125 (2011).

36 A. García-Etxarri, R. Gómez-Medina, L. S. Froufe-Pérez, C. López, L. Chantada, F. Scheffold, J. Aizpurua, M. Nieto-Vesperinas, and J. J. Sáenz, "Strong magnetic response of submicron Silicon particles in the infrared," Opt. Express 19, 4815-4826 (2011).

37 M. Nieto-Vesperinas, R. Gómez-Medina, and J. J. Sáenz, "Angle-suppressed scattering and optical forces on submicrometer dielectric particles," J. Opt. Soc. Am A 28, 54-60 (2011).

38 A. I. Kuznetsov, A. E. Miroshnichenko, Y. H. Fu, J. Zhang, and B. Luk'yanchuk, "Magnetic light," Sci. Rep. 2, 492 (2012).

39 Y. H. Fu, A. I. Kuznetsov, A. E. Miroshnichenko, Y. F. Yu, and B. Luk'yanchuk, "Directional visible light scattering by silicon nanoparticles," Nat. Commun. 4, 1527 (2013).

40 S. Person, M. Jain, Z. Lapin, J. J. Sáenz, G. Wicks, and L. Novotny, "Demonstration of zero optical backscattering from single nanoparticles," Nano Lett. 13, 1806-1809 (2013).

41 R. Paniagua-Domínguez, F. López-Tejeira, R. Marqués, and J. A. Sánchez-Gil, "Metallo-dielectric core-shell nanospheres as building blocks for optical threedimensional isotropic negative-index metamaterials," New J. Phys. 13, 123017 (2011).

42 R. Paniagua-Domínguez, D. R. Abujetas, and J. A. Sánchez-Gil, "Ultra low-loss, isotropic optical negativeindex metamaterial based on hybrid metal-semiconductor nanowires," Scientific Reports 3, 1507 (2013).

43 S. Ramadurgam and C. Yang, "Semiconductor-MetalSemiconductor Core-Multishell Nanowires as NegativeIndex Metamaterial in Visible Domain," Scientific Reports 4, 1 (2014).

44 W. Liu, A. E. Miroshnichenko, D. N. Neshev, and Y. S. Kivshar, "Broadband Unidirectional Scattering by Magneto-Electric Core-Shell Nanoparticles," ACS Nano 6, 5489-5497 (2012).

${ }^{45}$ W. Liu, J. Zhang, B. Lei, H. Ma, W. Xie, and H. Hu, "Ultra-directional forward scattering by individual coreshell nanoparticles," Opt. Express 22, 16178-16187 (2014).

46 R. Paniagua-Domínguez, D. R. Abujetas, L. S. FroufePérez, J. J. Sáenz, and J. A. Sánchez-Gil, "Broadband telecom transparency of semiconductor-coated metal nanowires: more transparent than glass," Opt. Express 21, 22076-22088 (2013).

47 W. Liu, A. E. Miroshnichenko, R. F. Oulton, D. N. Neshev, O. Hess, and Y. S. Kivshar, "Scattering of core-shell nanowires with the interference of electric and magnetic resonances," Opt. Lett. 38, 2621-2623 (2013).

48 W. Liu, J. Zhang, B. Lei, H. Hu, and A. E. Miroshnichenko, "Invisible nanowires with interfering electric and toroidal dipoles," Opt. Lett. 40, 2293-2296 (2015).

49 A. Poddubny, I. Iorsh, P. Belov, and Y. Kivshar, "Hyperbolic metamaterials," Nat. Photonics 7, 948-957 (2013).

${ }^{50}$ F. J. Valdivia-Valero and M. Nieto-Vesperinas, "Composites of resonant dielectric rods: A test of their behavior as metamaterial refractive elements," Photon. Nanostruct. Fundam. Applic. 10, 423-434 (2012).

51 S. Foteinopoulou and C. M. Soukoulis, "Negative refraction and left-handed behavior in two-dimensional photonic crystals," Phys. Rev. B 67, 235107 (2003).

52 J. L. García-Pomar and M. Nieto-Vesperinas, "Waveguiding, collimation and subwavelength concentration in photonic crystals," Opt. Express 13, 7997-8007 (2005). 
53 T. Decoopman, G. Tayeb, S. Enoch, D. Maystre, and B. Gralak, "Photonic crystal lens: From negative refraction and negative index to negative permittivity and permeability," Phys. Rev. Lett. 97, 073905 (2006).

54 A. L. Aden and M. Kerker, "Scattering of Electromagnetic Waves from Two Concentric Spheres," J. Appl. Phys. 22, 1242-1246 (1951).

55 X. Chen, T. M. Grzegorczyk, B. I. Wu, J. Jr. Pacheco, and J. A. Kong, "Robust method to retrieve the constitutive effective parameters of metamaterials," Phys. Rev. E 70, 016608 (2004).

56 D. R. Smith, D. C. Vier, T. Koschny, and C. M Soukoulis,
"Electromagnetic parameter retrieval from inhomogeneous metamaterials," Phys. Rev. E 71, 036617 (2005).

57 F. Dominec, C. Kadlec, H. Němec, P. Kužel, and F. Kadlec, "Transition between metamaterial and photoniccrystal behavior in arrays of dielectric rods," Opt. Express 22, 30492 (2014).

58 M. V. Rybin, I. S. Sinev, K. B. Samusev, A. Hosseinzadeh, G. B. Semouchkin, E. A. Semouchkina, and M. F. Limonov, "Photonic properties of two-dimensional high-contrast periodic structures: Numerical calculations," Phys. Solid State 56, 588-593 (2014).

59 E. D. Palik, Handbook of Optical Constants of Solids (Academic Press, 1998). 